\title{
Temperature measurements in a wall stabilized steady flame using CARS
}

\author{
Krishna $\mathrm{S}^{1}$, Deanna. A. Lacoste ${ }^{2}$ and William L. Roberts \\ and \\ Jason S. Damazo ${ }^{3}$ and Eddie Kwon ${ }^{4}$ \\ Boeing Research and Technology, Seattle, USA
}

Clean Combustion Research Center, King Abdullah University of Science and Technology, Saudi Arabia

\begin{abstract}
Flame quenching by heat loss to a surface continues to be an active area of combustion research. Close wall temperature measurements in an isothermal wall-stabilized flame are reported in this work. Conventional N2-vibrational Coherent Anti-Stokes Raman Scattering (CARS) thermometry as close as $275 \mu \mathrm{m}$ to a convex wall cooled with water has been carried out. The standard deviation of mean temperatures is observed to be $\sim 6.5 \%$ for high temperatures $(>2000 \mathrm{~K})$ and $\sim 14 \%$ in the lower range $(<500 \mathrm{~K})$. Methane/air and ethylene/air stoichiometric flames for various global strain rates based on exit bulk velocities are plotted and compared. $\mathrm{CH}^{*}$ chemiluminescence is employed to determine the flame location relative to the wall. Flame locations are shown to move closer to the wall with increasing strain rates in addition to higher near-wall temperatures. Peak temperatures for ethylene are considerably higher $(\sim 250-300 K)$ than peak temperatures for methane. Preheat zone profiles are similar for different strain rates across fuels. This work demonstrates close wall precise temperature measurments using CARS.
\end{abstract}

\section{Introduction}

$\mathrm{T}$ emperature measurements in a flame quenching environment provide critical information about the heat flux and the thermal environment close to the wall. Surface reactions also depend on the local temperature and could influence the radical quenching rates thereby affecting the quenching distance. The quenching Péclet number (Pe), is defined as the flame wall distance at quenching normalized by the flame thickness $\left(\delta_{\mathrm{F}}\right)$. For a methane air stoichiometric flame in a head-on quenching (HOQ) configuration the Pe is $\sim 1-1.5^{1}$ This means that quenching occurs at distances of a few millimeters from the wall. Therefore, precise measurements with high spatial resolution are essential in such configurations.

Numerous previous work has been carried out on stationary wall stabilized flames. Bergthorson $e t a .^{2}$ studied the hydrodynamics of stagnation flames experimentally and computationally. Their experimental setup consisted of a laminar jet flame impinging on a flat plate cooled by water. They observed that the axial velocity profile is independent of the nozzle to wall separation distance. While comparing simulations and experiments they report that the relative agreement is not affected by the imposed strain rate. Mann et al. ${ }^{3}$ studied a methane/air impinging jet flame on a water-cooled stainless steel wall. Temperatures were measured using broadband $\mathrm{N}_{2}$ vibrational CARS while CO concentrations were obtained from PLIF measurements. Both stationary flames and transient quenching were studied. Wall temperatures were measured using phosphor thermometry. They observed that the quenching distances are in the range of 0.17-0.32 $\mathrm{mm}$ while the wall heat flux during quenching is twice the heat flux at

${ }^{1}$ Post-Doctoral Research Fellow, Clean Combustion Research Center, King Abdullah University of Science and Technology, Saudi Arabia

${ }^{2}$ Assistant Professor, Clean Combustion Research Center, King Abdullah University of Science and Technology, Saudi Arabia

${ }^{3}$ Physicist, Boeing Research and Technology, Seattle, USA.

${ }^{4}$ Physicist, Boeing Research and Technology, Seattle, USA.

${ }^{5}$ Professor and Director, Clean Combustion Research Center, King Abdullah University of Science and Technology, Saudi Arabia

American Institute of Aeronautics and Astronautics 
stationary conditions. Singh et al. ${ }^{4}$ observed that changing the wall material to quartz does not modify the peak temperature or $\mathrm{CO}$ levels for the same conditions. Bohlin et al. ${ }^{5}$ studied measured temperatures using a two beam rotational CARS system in the same configuration. They were able to obtain a very high spatial resolution of $\sim 61$ $\mu \mathrm{m}$ with close wall single-shot temperature measurements. Bouvet $e t$ al. ${ }^{6}$ carried out $1 \mathrm{D}$ and $2 \mathrm{D}$ simulations using Fluent 6.3 for a round jet methane/air stoichiometric flame impinging on an insulated metal plate and compared computed velocity field with PIV measurements. They concluded that 1D simulations fail to capture the strong pressure variations due to the nozzle-wall proximity and that 2D simulations that account for the effect of particle slip and thermophoresis are better at predicting the velocities.

This work uses precise temperature measurements to examine the effect of strain rate and fuel on flame quenching. Past works in a stagnation flame stabilized on an isothermal wall have utilized laser diagnostic techniques primarily due to restrictions of near-wall measurements. Thermocouple measurements are restricted by the bead size and radiation from the flame and the wall affecting the temperature measurements. Laser sheet based measurement techniques are not suitable for close wall measurements due to reflections from the surface. Coherent Anti Stokes Raman Scattering (CARS) is a technique that has an advantage in such geometries due to the small probe volumes employed. Temperature measurements as close as $100 \mu \mathrm{m}^{7}$ and $25 \mu \mathrm{m}^{3}$ have been reported. This work demonstrates the capability of the CARS system to measure temperatures sufficiently close to a wall under steady state conditions relevant to flame quenching. Although flame quenching is an inherently transient phenomenon, the current study is of importance owing to the close wall measurement capabilities and the effect of strain rates on the flame stabilization.

\section{Experimental setup}

The wall stabilized flame configuration is similar to the one employed by Mann et al. ${ }^{3}$ shown in Fig 1.
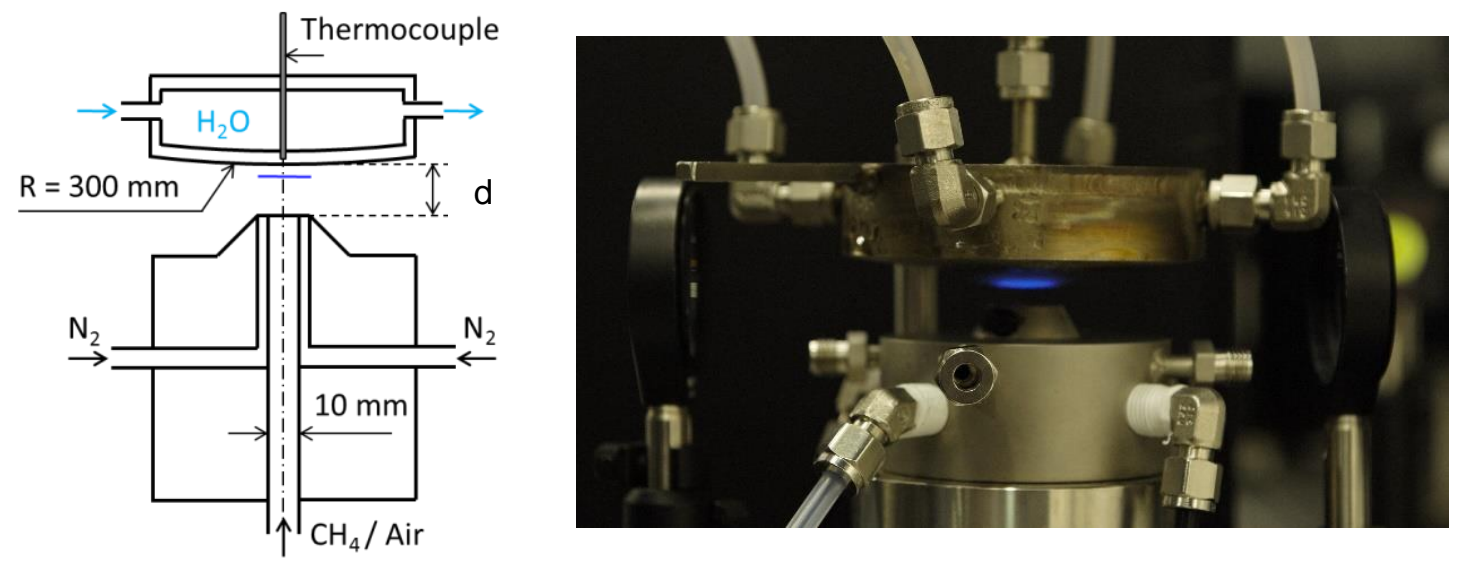

Figure 1. Schematic of burner wall assembly (left). Steady flame stabilized close to burner wall (right).

A premixed laminar burner with a central flow tube of $10 \mathrm{~mm}$ diameter is used with a co-flow of nitrogen to prevent dilution from surrounding air. The burner consists of a contoured nozzle to obtain flat velocity profile at the exit. Fuel and air flow metering is achieved using mass flow controllers (Brooks Instruments). Nitrogen co-flow is manually controlled to ensure an undisturbed flame. A specially designed stainless steel wall with a thickness of 5 $\mathrm{mm}$ is placed at a distance of $d(\mathrm{~mm})$ from the top of the burner. The wall has a convex profile to avoide beam clipping at close-wall measurement locations. The wall is cooled using water circulated through radially opposing inlets and outlets. The temperature of the wall is measured using a thermocouple inserted into the wall from the top. Methane-air and ethylene-air premixtures at an equivalence ratio $\varphi=1$ with a bulk exit velocity, $U_{b u l k}(\mathrm{~m} / \mathrm{s})$ varied relative to the laminar burning velocity $\left(S_{L}\right)$ is supplied. Consequently, the flames stabilize at different locations. Global strain rate, $a\left(\mathrm{~s}^{-1}\right)$, is defined to characterize the flame based on $U_{b u l k}$ and $d$ as follows

$$
a=\frac{U_{b u l k}}{d}
$$


The wall and the burner are mounted on a single vertical translation stage to measure at different locations. The separation distance, $d$, is fixed at $9 \mathrm{~mm}$, based on the optimum CARS signal intensity with miminum beam clipping while the $U_{\text {bulk }}$ is varied to attain flame stabilization at various strain rates.

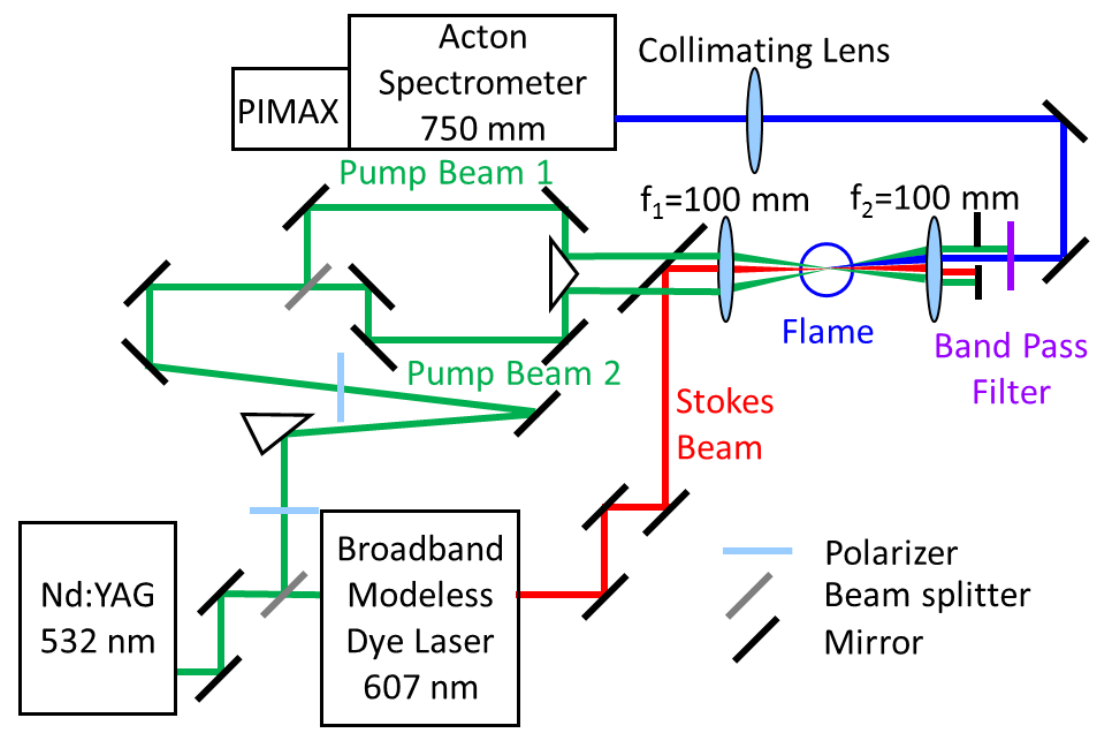

Figure 2. Schematic of Q-branch vibrational $\mathrm{N}_{2}$ CARS system.

In the present study, a $\mathrm{N}_{2}$ Q-branch vibrational CARS system has been implemented, a schematic of which is shown in Fig. 2. The pump and probe beams at $532 \mathrm{~nm}$ are provided by the $2^{\text {nd }}$ harmonic of a Spectra Physics Quanta Ray Nd:YAG laser. The Stokes beam is obtained by stimulated fluorescence of Sulforhodamine 640 in methanol solvent with a concentration of $40 \mathrm{mg} / \mathrm{L}$. The dye laser has a modeless broadband emission centered at $\sim 607 \mathrm{~nm}$. The three beams are focused using a plano-convex lens resulting in a probe volume of dimension $650 \mu \mathrm{m}$ in the direction of propagation of the beams and $30 \mu \mathrm{m}$ diameter at the focal point. A folded-BOXCARS alignment is employed wherein the pump and probe beams lie in one plane while the Stokes and CARS beams lie in another. This configuration is known to provide excellent spatial resolution and clear separation of beams albeit with lower signal strengths (Muller et al.). The path lengths of the pump and probe beams are adjusted in such a way that they are same as that of the Stokes beam. The beam energies of the $532 \mathrm{~nm}$ beams are $\sim 18 \mathrm{~mJ} / \mathrm{pulse}$ whereas the $607 \mathrm{~nm}$ beam has an energy of $\sim 3 \mathrm{~mJ} /$ pulse. The spatial resolution is measured using a cover slip mounted on a linear translation stage. The CARS signal is then collected and sent through a bandpass filter centered at $473 \mathrm{~nm}$ with a full width at half maximum (FWHM) of $10 \mathrm{~nm}$ and a collimating lens with focal length $100 \mathrm{~mm}$ is used to focus the beam at the slit of the spectrometer which has an opening of $100 \mu \mathrm{m}$. A spectrometer (Acton, $\mathrm{f}=750 \mathrm{~mm}, 1800$ lines/mm grating) equipped with an ICCD camera (PIMAX-3, Princeton Instruments, 1024 x 256 resolution) is used to collect and record the spectra. The camera was gated at $100 \mathrm{~ns}$ while the gain was maintained at a minimum of 1 to reduce the noise. A non-resonant background signal is obtained from an argon environment. 

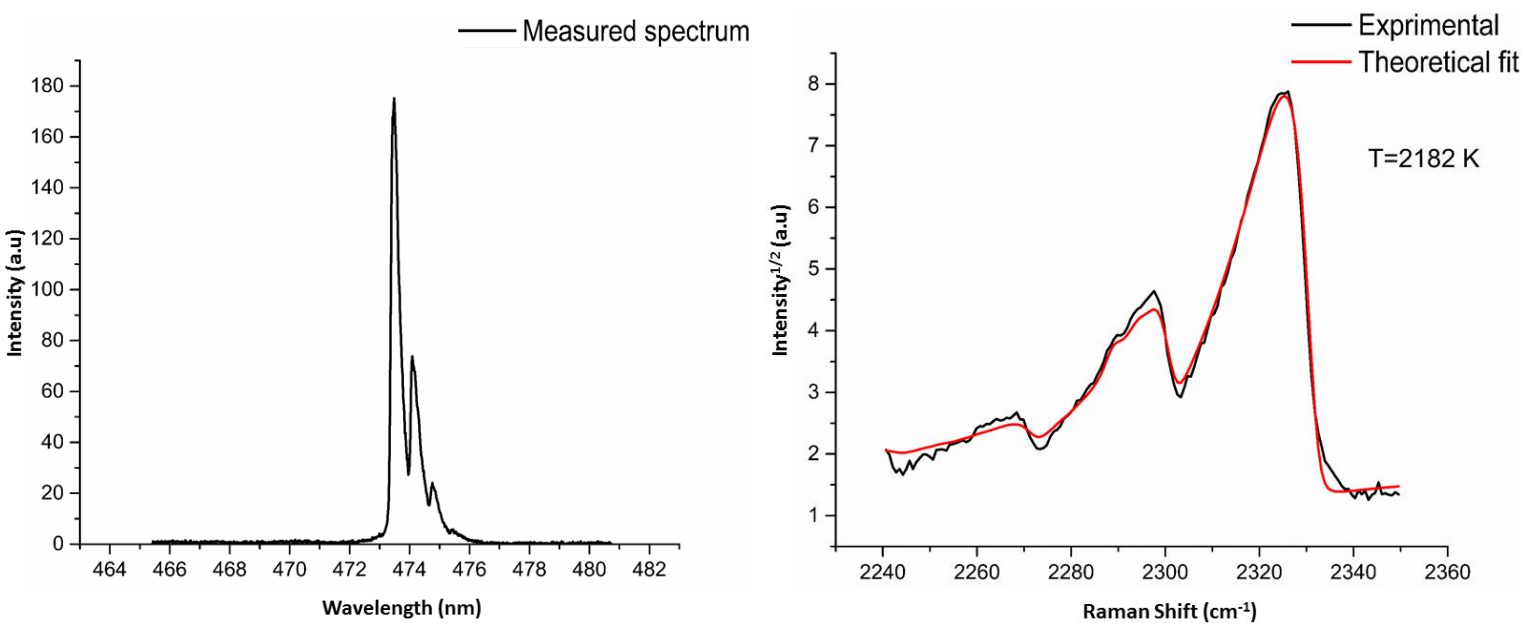

Figure 3. Typical measurement (left) and fitted CARS spectrum (right)

The obtained spectra are averaged and corrected for background noise. The signal is then divided by the nonresonant background signal and the Raman shift is applied to obtain the CARS spectra. The temperature at the probe volume is estimated by fitting the obtained CARS spectra with theoretical spectra for $\mathrm{N}_{2}$. The CARSFT ${ }^{8}$ code is used for the least square fitting. A typical fitting of CARS spectrum for high temperature ( $\mathrm{T}=2182 \mathrm{~K})$ is shown in Fig 3. Temperatures at different axial locations along the centerline from the wall to burner exit were measured at $100 \mu \mathrm{m}$ intervals. The flame contour follows the shape of the wall and hence compared to the probe volume dimension $(<1 \mathrm{~mm})$, curvature at the center can be neglected and the flame assumed to be flat. Mean spectra from 500 single shots are reported. An uncertainty analysis was carried out to determine the precision of the measurements. The standard deviations of 10 single shots from the mean were calculated for high temperature (> $2000 \mathrm{~K}$ ) and lower temperatures $(<500 \mathrm{~K})$ and found to be $6.5 \%$ and $14 \%$ respectively.

Flame location is determined by using $\mathrm{CH}^{*}$ chemiluminescence. $\mathrm{CH}^{*}$ radicals are produced in the region close to the high temperature reaction zone in hydrocarbon flames, with characteristic emission $\sim 432 \mathrm{~nm}$. An ICCD camera (PIMAX-3, Princeton Instruments) mounted with a UV lens and a broadband interference filter centered at $430 \mathrm{~nm}$ and with a FWHM of $10 \mathrm{~nm}$ is used to obtain the chemiluminescence signal. The camera exposure was set at $500 \mu$ s with a gain of 1 Figure 4 shows the $\mathrm{CH}^{*}$ chemiluminescence images for a $=220 \mathrm{~s}^{-1}$ with methane and ethylene fuels The peak $\mathrm{CH}^{*}$ emission location is determined and is matched with the spatial location of temperature that equals 0.9 times the peak value ( $\mathrm{T}_{\text {peak }}$ ) based on Blevins et al. ${ }^{9}$ and $\mathrm{Li}$ et al. ${ }^{10}$. The accuracy of the flame location is about $15 \mu \mathrm{m}$.
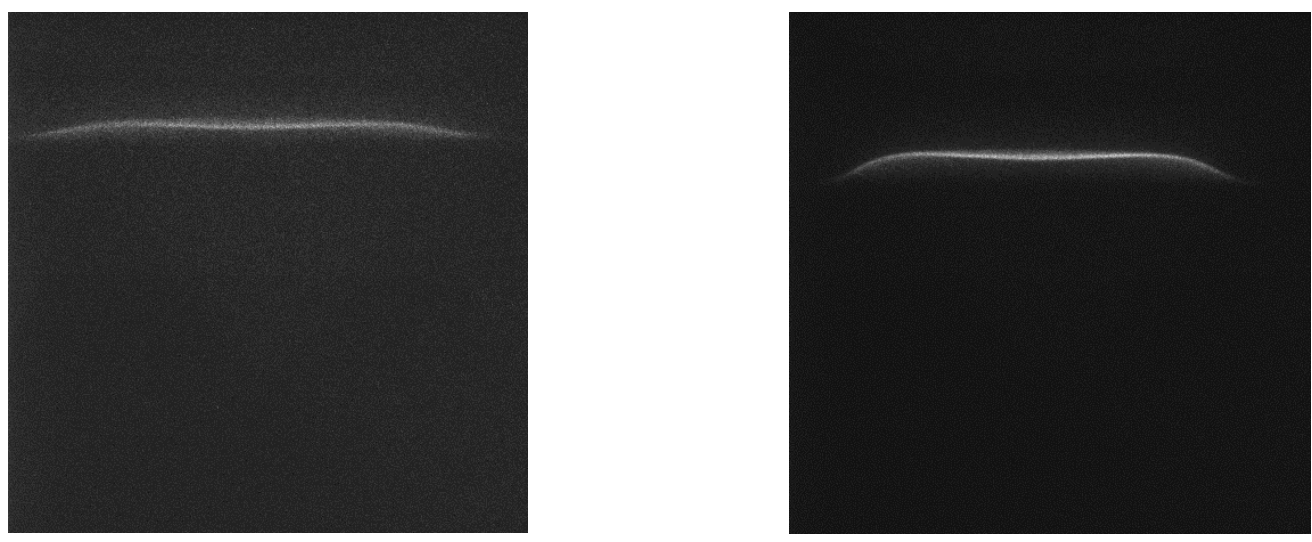

Figure 4. $\mathrm{CH}^{*}$ chemiluminescence for strain rate $a=220 \mathrm{~s}^{-1}$ for stoichiometric methane-air (left) and ethylene-air (right) flames 


\section{Results and discussion}

Measured temperature data from CARS are reported as axial centerline profiles at different global strain rates. Figure 5 shows the comparison of temperature profiles for three different strain rates for methane-air stoichiometric flames. The black symbols on Fig. 5 show such a profile for $\mathrm{CH}_{4} /$ air stoichiometric mixture with $a=135 \mathrm{~s}^{-1}$ corresponding to $U_{b u l k}=3 S_{L}$. It can be seen that the temperature near the wall is lower than the peak temperature of around $2100 \mathrm{~K}$ due to heat transfer to the water-cooled wall. The peak temperature is also lower than the adiabatic flame temperature for methane-air at $\varphi=1(2222 \mathrm{~K})$. The closest temperature measurement location was determined to be $275 \mu \mathrm{m}$ from the wall. The wall temperature is $328 \mathrm{~K}$ for this condition. This indicates a steep gradient in the region between the closest measured location and the wall. The preheat zone temperature gradient is seen to consist of two slopes, namely a flatter gradient from $2.25 \mathrm{~mm}$ to $3.25 \mathrm{~mm}$ and a steeper one from $3.25 \mathrm{~mm}$ to $3.75 \mathrm{~mm}$. This has been observed in previous works $\mathrm{s}^{3,11,12}$ and is characteristic of premixed laminar flames.

The effect of the strain rates on the flame location can be clearly observed from the temperature profiles in Fig. 5. The peak temperature region moves closer to the wall with increasing strain rates and decreases in thickness by as much as $128 \%$ when the strain rate is decreased from $220 \mathrm{~s}^{-1}$ to $135 \mathrm{~s}^{-1}$. The preheat zone which corresponds to the location of the visible flame shifts by close to $1 \mathrm{~mm}$ when the strain rate increases to $175 \mathrm{~s}^{-1}$. In addition the flame thickness is reduced due to the stretching of the flame due to the increased velocity and the stagnation point flow near the wall. Consider the case of $\mathrm{U}_{\text {bulk }}=5 \mathrm{~S}_{\mathrm{L}}$, corresponding to $\mathrm{a}=220 \mathrm{~s}^{-1}$, where the temperatures are lower than for the case with $\mathrm{a}=135 \mathrm{~s}^{-1}$. This is due to the heat transfer from the flame to the wall. It must also be noted that the temperatures fall within the uncertainty limit of measurements owing to the CARS technique. It is also worthwhile to observe that the temperature gradient in the preheat zone is similar across various strain rates. This can be attributed to the presence of two slopes in the preheat zone. The steeper gradient is observed between $1800 \mathrm{~K}$ and $300 \mathrm{~K}$ irrespective of the peak temperatures and hence does not depend on the location of the flame or the strain rates. However the flatter gradient between the peak temperature and $1800 \mathrm{~K}$ varies based on the flame location.

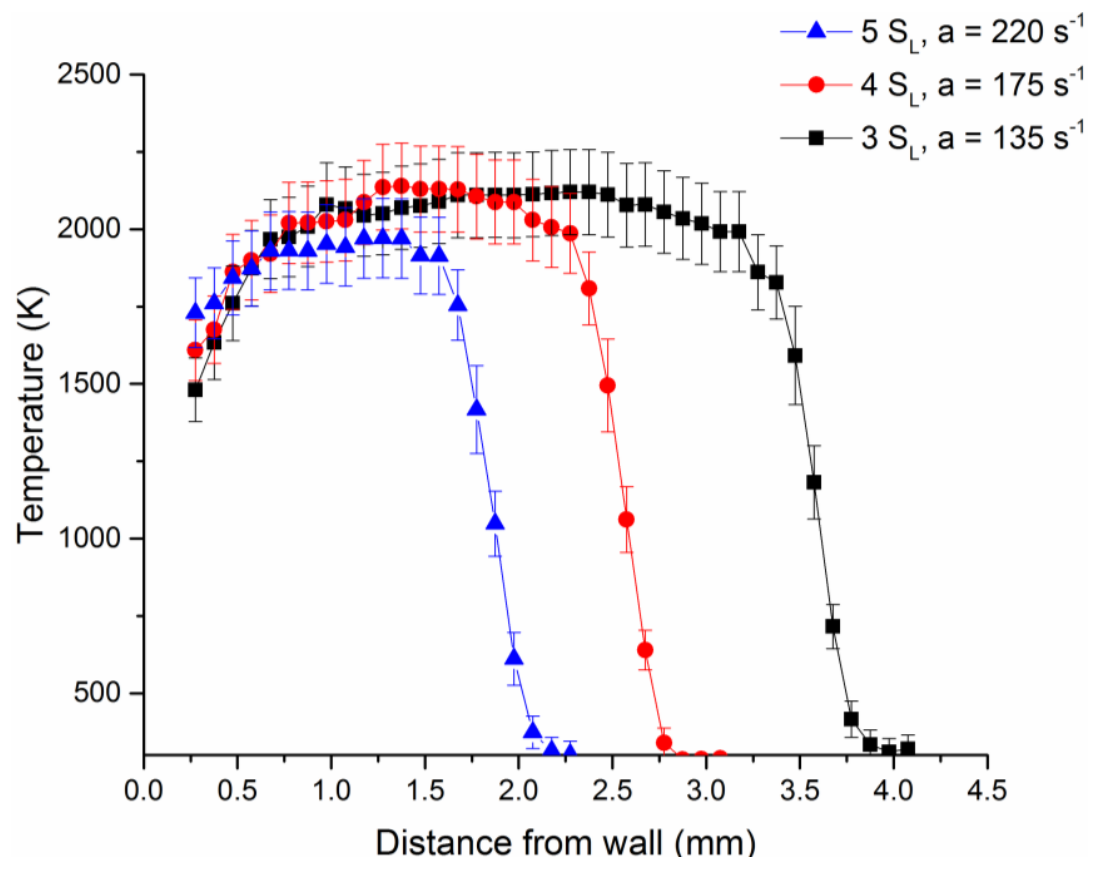

Figure 5. Axial centerline temperature profile for stoichiometric methane-air flames at three strain rates

The effect of the fuel on the flame stabilization and temperatures has been investigated through ethylene/air stoichiometric flames, stabilized in the same configuration. It is important to note here that the strain rates were held constant in order to provide a basis for comparing the effect of the fuel. Figure 6 compares the temperature profiles for methane and ethylene at a strain rate of $a=220 \mathrm{~s}^{-1}$. Two major observations can be made frome the plot in Fig 5. First, the location of the flame for the same strain rate is considerably farther from the wall in the case of ethylene. This is also seen in $\mathrm{CH}^{*}$ chemiluminescence imaging as shown in Fig 4. This can be explained on the basis of the 
laminar flame velocities. The $S_{L}$ for stoichiometric ethylene-air flames at atmospheric pressure is $\sim 70 \mathrm{~m} / \mathrm{s}$ which is almost twice the laminar flame speed for methane-air stoichiometric flames. Hence for the same exit velocities of fuel-air premixture, the flame is stabilized farther away from the wall in the case of ethylene.

The second observation is related to the higher peak temperatures for the ethylene flame. This can be attributed to the fact that ethylene-air stoichiometric flames have an adiabatic flame temperature of about $2600 \mathrm{~K}$. Compared to methane, the flame temperature is about $400 \mathrm{~K}$ higher and this is clearly reflected in the difference in measured peak temperatures. In spite of the higher peak temperature in the case of ethylene, the temperature measured at 275 $\mu \mathrm{m}$ close to the wall is lower than in the case of methane due to the flame location being considerably farther than methane. The preheat zone clearly shows two slopes in the case of ethylene which, as discussed before, is due to higher peak temperature. It is also interesting to note that the steeper slope begins at $1900 \mathrm{~K}$.

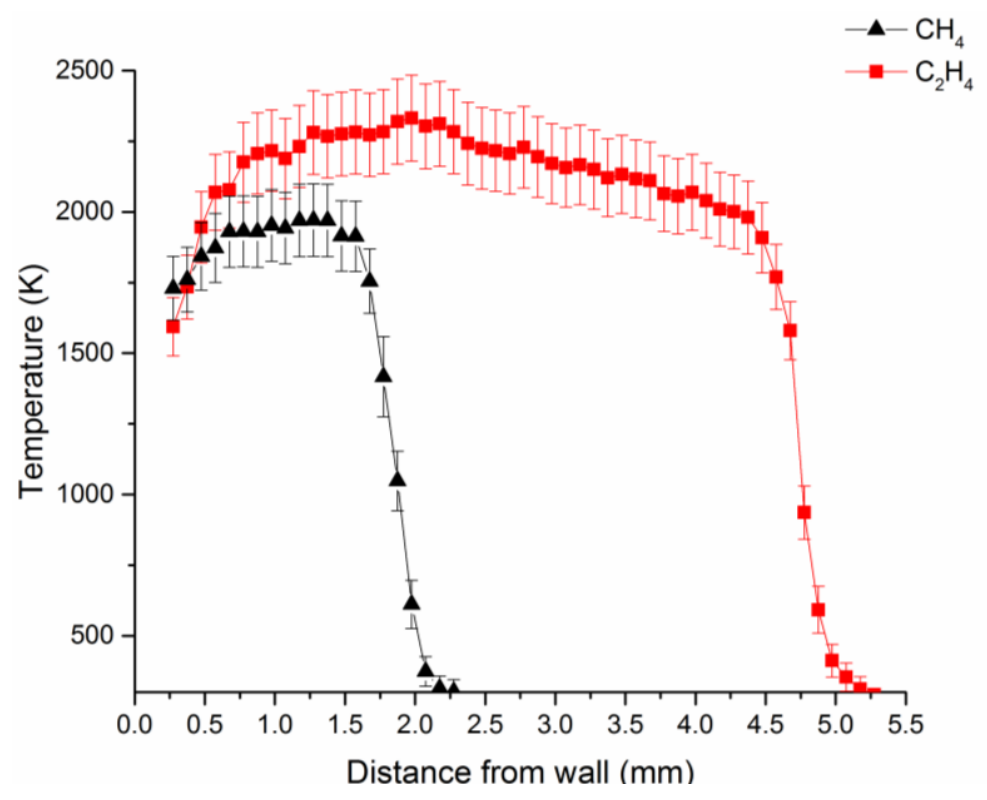

Figure 6. Axial centerline temperature profile for stoichiometric methane- and ethylene-air flames with strain rate $a=220 \mathrm{~s}^{-1}$

\section{Conclusion}

A $\mathrm{N}_{2}$ Q-branch vibrational CARS thermometry system has been implemented and preliminary measurements as close as $\sim 275 \mu \mathrm{m}$ to an isothermal wall are reported. $\mathrm{CH}^{*}$ chemiluminescence was employed to precisely locate the flame front relative to the wall. Temperature profiles for methane-air stoichiometric wall stabilized flames at various strain rates show a marked change in the flame location. The measured peak tempeartures and gradients have been explained based on this. Ethylene-air stoichiometric flame at the same strain rate has also been studied and the observed increase in peak temperature is attributed to the higher adiabatic flame temperature. In addition, the presence of two distinct slopes in the preheat zone has been observed both in the case of methane and ethylene. This is in line with previous works in literature. Further work will involve numerical simulations to compare with experiments.

\section{Acknowledgments}

This work has been partly funded by The Boeing Company and the King Abdullah University of Science and Technology.

\section{References}

${ }^{1}$ Dreizler, A., Böhm, B., "Advanced laser diagnostics for an improved understanding of premixed flame-wall interactions," Proceedings of the Combustion Institute, Vol. 35, 2015, pp. 37-64 
${ }^{2}$ Bergthorson, J. M., Salusbury, S. D., and Dimotakis, P. E., "Experiments and modelling of premixed laminar stagnation flame hydrodynamics," J. Fluid Mech., vol. 681, 2011, pp. 340-369

${ }^{3}$ Mann, M., Jainski, C., Euler, M., Böhm, B., Dreizler, A., "Transient flame-wall interactions: Experimental analysis using spectroscopic temperature and CO concentration measurements," Combustion and Flame, Vol. 161, 2014, pp. 2371-2386

${ }^{4}$ Singh, A., Mann, M., Kissel, T., Brübach, J., Dreizler, A., "Simultaneous Measurements of Temperature and CO Concentration in Stagnation Stabilized Flames," Flow Turbulence Combust, Vol. 90, 2013, pp. 723-739

${ }^{5}$ Bohlin, A., Mann, M., Patterson, B. D., Dreizler, A., Kliewer, C. J., "Development of two-beam femtosecond/picosecond one-dimensional rotational coherent anti-Stokes Raman spectroscopy: Time-resolved probing of flame wall interactions," Proceedings of the Combustion Institute, Vol. 35, 201, pp. 3723-3730

${ }^{6}$ Bouvet, N., Davidenko, D., Chauveau, C., Pillier, L., Yoon, Y., "On the simulation of laminar strained flames in stagnation flows: 1D and 2D approaches versus experiments," Combustion and Flame, Vol. 161, 2014, pp. 438-452

${ }^{7}$ Lucht, R., Maris, M., "CARS Measurements of Temperature Profiles Near a Wall in an Internal Combustion Engine," SAE Technical Paper, 870459, 1987, doi:10.4271/870459

${ }^{8}$ Palmer, R. E., The CARSFT computer code for calculating coherent anti-stokes Raman spectra: user and programmer information, Sandia National Laboratories Report SAND89-8206, Livermore, CA, 1989

${ }^{9}$ Blevins, L. G., Renfro, M. W., Lyle, K. H., Laurendeau, N. M., Gore, J. P., "Experimental study of temperature and CH radical location in partially premixed $\mathrm{CH}_{4}$ /Air coflow flames," Combustion and Flame, Vol. 118, 1999, pp. 684-696

${ }^{10} \mathrm{Li}, \mathrm{T}$., Adamovich I. V., Sutton, J. A., "Effects of non-equilibrium plasmas on low-pressure, premixed flames. Part 1: CH* chemiluminescence, temperature, and $\mathrm{OH}$," Combustion and Flame, Vol. 165, 2016, pp. 50-67

${ }^{11}$ Delfau, J-L., Biet, J., Idir, M., Pillier, L., Vovelle, C., "Experimental and numerical study of premixed, lean ethylene flames," Proceedings of the Combustion Institute, Vol. 31, Issue 1, 2007, pp. 357-365

${ }^{12}$ Biet, J., Delfau, J-L., Seydi, A., Vocelle, C., "Experimental and modeling study of lean premixed atmospheric-pressure propane/O2/N2 flames," Combustion and Flame, Vol. 142, Issue 3, 2005, pp. 197-209 